\title{
Dimensional effects on the momentum distribution of bosonic trimer states
}

\author{
F. F. Bellotti, ${ }^{1,2,4}$ T. Frederico, ${ }^{1}$ M. T. Yamashita, ${ }^{3}$ D. V. Fedorov, ${ }^{4}$ A. S. Jensen, ${ }^{4}$ and N. T. Zinner ${ }^{4}$ \\ ${ }^{1}$ Instituto Tecnológico de Aeronáutica, 12228-900 São José dos Campos, SP, Brazil \\ ${ }^{2}$ Instituto de Fomento e Coordenação Industrial, 12228-901 São José dos Campos, SP, Brazil \\ ${ }^{3}$ Instituto de Física Teórica, UNESP-Univ Estadual Paulista, Caixa Postal 70532-2, CEP 01156-970 São Paulo, SP, Brazil \\ ${ }^{4}$ Department of Physics and Astronomy, Aarhus University, DK-8000 Aarhus C, Denmark
}

(Received 23 July 2012; published 11 January 2013)

\begin{abstract}
The momentum distribution is a powerful probe of strongly interacting systems that are expected to display universal behavior. This is contained in the contact parameters which relate few- and many-body properties. Here we consider a Bose gas in two dimensions and explicitly show that the two-body contact parameter is universal and then demonstrate that the momentum distribution at next-to-leading order has a logarithmic dependence on momentum which is vastly different from the three-dimensional case. Based on this, we propose a scheme for measuring the effective dimensionality of a quantum many-body system by exploiting the functional form of the momentum distribution.
\end{abstract}

DOI: 10.1103/PhysRevA.87.013610

PACS number(s): 67.85.-d, 21.45.-v, 36.40.-c

\section{INTRODUCTION}

Strongly interacting quantum systems are ubiquitous in nature and naturally at the forefront of physics research. However, the theoretical study of strong interactions can be very difficult since our usual and intuitively clear perturbative methods can fail miserably when interparticle forces are strong. A success of cold atomic gas physics is the ability to create and manipulate strongly interacting gases in experiment [1]. One particularly interesting aspect is the ability to change the dimensionality by applied optical fields. This means that low-dimensional dynamics which is typically found in condensed-matter systems of great technological interest can be addressed. The field thus provides a test bed for models of strongly coupled dynamics that are used to describe interesting materials.

A breakthrough in the study of strongly interacting quantum systems with short-range interactions was the derivation of a set of universal relations that relate the two-body correlations to the many-body thermodynamics through the so-called contact parameter $C_{2}$ [2]. One way to define this quantity is through the asymptotic behavior of the single-particle momentum distribution $n(\boldsymbol{k})$ of a few- or many-body system, i.e., via $n(\boldsymbol{k}) \rightarrow C_{2} / k^{4}$ which is the leading-order behavior when the momentum $\boldsymbol{k}$ goes to infinity (this fact had been already derived for a one-dimensional Bose gas with zerorange interactions, the Lieb-Liniger system [3]). The same $C_{2}$ also appears in the total energy of the system and in response functions. These relations were subsequently confirmed in experiments on two-component Fermi gases [4,5]. They also hold for bosonic gases [6-10] as confirmed by recent experiments [11]. In the case of two-component fermions, the Pauli principle suppresses correlations between three particles. In contrast, for bosons three-body correlations are very important and this implies that one must also consider a three-body contact parameter $C_{3}[8,9]$. It is most simply defined as the coefficient of the subleading large $\boldsymbol{k}$ limiting term in $n(\boldsymbol{k})$, but as we will show below, the form of this term is highly sensitive to dimensionality.

A second avenue that is enjoying great success at the moment, is the experimental study of two-dimensional (2D) atomic Fermi gases [12-16]. Universal contact relations should also hold in this case [6,17-20]. Interestingly, a recent experiment [21] has found that the monopole breathing mode is essentially undamped and has no interaction-dependent shift [22-24]. This implies a scale invariance in the system [25] that has also been observed in weakly interacting $2 \mathrm{D}$ Bose gases [26]. However, this observation is hard to reconcile with the fact that a scale is provided by the energy of the two-body dimer which is always bound for attractive short-range interaction in 2D. One would naively expect modifications of both few- and many-body dynamics in these systems.

From a few-body perspective, the special features of 2D systems are manifest in the spectrum of three identical bosons with attractive zero-range interactions (the so-called universal limit), since no length scale is provided by the two-body potential except for the one given by the two-body dimer binding energy $E_{2}$. Here one finds that there are exactly two bound states which have energies $E_{3}=16.52 E_{2}$ and $E_{3}=$ $1.270 E_{2}[27-30]$. This is in sharp contrast to three-dimensional (3D) systems, where an infinite set of geometrically separated states appear at the threshold for two-body binding [31]. In realistic systems, this scaling is broken by the finite range of the interaction [32], and one obtains a normalization of the spectrum since the range determines the lowest bound universal bound state (there are deeply bound states that have small radii and nonuniversal structure which are not of interest here). Typically one parametrizes the short-range physics by introducing the three-body parameter $\kappa_{*}^{3 \mathrm{D}}$ to get the correct three-body energy [32]. However, in 2D such a procedure is not needed for three particles, i.e., there is no need for a $\kappa_{*}^{2 \mathrm{D}}$. In the universal limit in 2D this implies that the three-body energies must be proportional to the dimer energy.

In this paper, we study identical bosons in $2 \mathrm{D}$ with attractive short-range interactions and use few-body methods to determine $C_{2}$ and $C_{3}$. This is achieved by computing the momentum distribution for three identical bosons, in particular, its asymptotic behavior for large momenta. We provide both analytical and numerical evidence that support a universal tail behavior that is the same for both ground and excited states. To the best of our knowledge, $C_{3}$ has not 
been discussed in 2D before. Moreover, we show that the subleading term has a novel behavior that is radically different in $2 \mathrm{D}$ as compared to $3 \mathrm{D}$. Based on this fact, we propose to use the momentum distribution to measure the effective dimensionality of a quantum system in the universal regime. Our study is thus a first step in exploring effects of dimensional crossover on higher-order correlations in many-body systems.

\section{METHOD}

We consider three identical bosons with mass $m$. We use attractive two-body interactions of zero range and parametrized by the dimer binding energy $E_{2}$. The two-body $T$ matrix for energy $E$ is thus $\tau(E)=\left(-2 \pi \ln \sqrt{-E / E_{2}}\right)^{-1}$ in units where $\hbar=m=1[28,33,34]$. By using Faddeev decomposition and bosonic symmetry, the three-body wave function $\Psi$ can be written

$$
\Psi(\mathbf{q}, \mathbf{p})=\frac{f(q)+f\left(\left|\mathbf{p}-\frac{\mathbf{q}}{2}\right|\right)+f\left(\left|\mathbf{p}+\frac{\mathbf{q}}{2}\right|\right)}{E_{3}+\mathbf{p}^{2}+\frac{3}{4} \mathbf{q}^{2}},
$$

where $\boldsymbol{p}=\frac{1}{2}\left(\boldsymbol{k}_{1}-\boldsymbol{k}_{2}\right)$ and $\boldsymbol{q}=\frac{2}{3} \boldsymbol{k}_{3}-\frac{1}{3}\left(\boldsymbol{k}_{1}+\boldsymbol{k}_{2}\right)$ are Jacobi momenta, $\boldsymbol{k}_{i}, i=1,2,3$ are the laboratory momenta, and $E_{3}$ is the three-body energy. The spectator functions $f(\boldsymbol{q})$ satisfy the set of integral equations

$$
f(\mathbf{q})=2 \tau\left(-E_{3}-\frac{3}{4} \mathbf{q}^{2}\right) \int d^{2} k \frac{f(\mathbf{k})}{-E_{3}-\mathbf{q}^{2}-\mathbf{k}^{2}-\mathbf{k} \cdot \mathbf{q}} .
$$

Armed with the solution to this equation, the momentum distribution is

$$
n(q)=\int d^{2} p\left|\frac{f(q)+f\left(\left|\mathbf{p}-\frac{\mathbf{q}}{2}\right|\right)+f\left(\left|\mathbf{p}+\frac{\mathbf{q}}{2}\right|\right)}{E_{3}+\mathbf{p}^{2}+\frac{3}{4} \mathbf{q}^{2}}\right|^{2} .
$$

Following the discussion in Ref. [9], we define four components $n^{m}(q)=\sum_{i=1}^{4} n_{i}^{m}(q)$, where $m=0$ denotes the ground state and $m=1$ the excited state. The individual components are

$$
\begin{gathered}
n_{1}^{m}(q)=f_{m}^{2}(q) \int d^{2} p \frac{1}{\left(E_{3}^{m}+\mathbf{p}^{2}+\frac{3}{4} \mathbf{q}^{2}\right)^{2}}=\frac{\pi f_{m}^{2}(q)}{E_{3}^{m}+\frac{3}{4} \mathbf{q}^{2}} \\
n_{2}^{m}(q)=4 f_{m}(q) \int d^{2} k \frac{f_{m}(k)}{\left(E_{3}^{m}+\mathbf{k}^{2}+\mathbf{q}^{2}+\mathbf{k} \cdot \mathbf{q}\right)^{2}} \\
n_{3}^{m}(q)=2 \int d^{2} k \frac{f_{m}^{2}(k)}{\left(E_{3}^{m}+\mathbf{k}^{2}+\mathbf{q}^{2}+\mathbf{k} \cdot \mathbf{q}\right)^{2}} \\
n_{4}^{m}(q)=2 \int d^{2} k \frac{f_{m}(k) f_{m}(|\mathbf{k}-\mathbf{q}|)}{\left(E_{3}^{m}+\mathbf{k}^{2}+\mathbf{q}^{2}+\mathbf{k} \cdot \mathbf{q}\right)^{2}}
\end{gathered}
$$

where $m$ on $f(q)$ and $E_{3}$ labels the state. Throughout, we measure all momenta in units of $\sqrt{E_{2}}$. Note that the normalization we use is $\int d^{2} k n(k)=1$.

\section{LARGE-MOMENTUM LIMIT}

The leading-order (LO) behavior of the momentum distribution exhibits the same $C_{2} k^{-4}$ tail in 1D, 2D, and 3D since it derives solely from two-body physics [35]. However, $C_{2}$ depends on what system is addressed and whether few-body bound states are present. For bosons in $3 \mathrm{D}$, the tail is [8,9]

$$
n_{3 \mathrm{D}}(k) \rightarrow \frac{1}{k^{4}} C_{2}+\frac{\cos \left[2 s_{0} \ln \left(\sqrt{3} k / \kappa_{*}\right)+\phi\right]}{k^{5}} C_{3},
$$

where $s_{0}=1.00624$ and $\phi=-0.87280$ are constants that can be determined from a full solution of the three-bosons problem in 3D at unitarity [9] with trimer energy $E_{3}=\kappa_{*}^{2}$ (using $\kappa_{*}^{3 \mathrm{D}}=$ $\kappa_{*}$ for simplicity). The log-periodic three-body next-to-leading order (NLO) term derives from the Efimov effect, whose solution can be used to determine $3(2 \pi)^{3} C_{2}=53.097 / \kappa_{*}$ and $3(2 \pi)^{3} C_{3}=-89.263 / \kappa_{*}^{2}[9]$. The factor $3(2 \pi)^{3}$ is due to a difference in definition of $n(k)$ in Eq. (3) in comparison to Ref. [9]. As discussed above, in 2D there is no Efimov effect for three bosons. The log-periodic behavior is therefore not expected a priori. As we will now demonstrate, the distribution in $2 \mathrm{D}$ is very different. It has the structure

$$
n_{2 \mathrm{D}}(k) \rightarrow \frac{1}{k^{4}} C_{2}+\frac{\ln ^{3}(k)}{k^{6}} C_{3},
$$

and we see indeed a very different NLO term. We note that the NLO term is different from the fermionic case discussed in Ref. [17] where no $\ln (k)$ factors are present and implies that quantum statistics plays a role in determining the functional form of the NLO term. Furthermore, it implies that the NLO term is in fact an effective measure of dimensionality of bosonic systems in the universal regime. We will return to this point below.

To derive the tail behavior in Eq. (9), one needs to determine first the spectator function $f_{m}(\boldsymbol{q})$ in Eq. (2) for large $q$. This can be done analytically and we provide the details in Appendix A. The result is that $f_{m}(q) \rightarrow A_{m} \ln (q) / q^{2}$, where $A_{m}$ is a statedependent constant. This function can now be inserted into Eqs. (4)-(7) and the momentum tail can be determined. The technical details are given in Appendix B. After the dust settles, the tail behaviors can be written

$$
\begin{aligned}
& n_{1}^{m}(q) \rightarrow \frac{4 \pi}{3} \frac{A_{m}^{2} \ln ^{2}(q)}{q^{6}}, \quad n_{2}^{m}(q) \rightarrow 4 \pi \frac{A_{m}^{2} \ln ^{3}(q)}{q^{6}}, \\
& n_{3}^{m}(q) \rightarrow \frac{4 \pi}{q^{4}} \int_{0}^{\infty} d k k f_{m}^{2}(k), \quad n_{4}^{m}(q) \rightarrow 2 \pi \frac{A_{m}^{2} \ln ^{3}(q)}{q^{6}} .
\end{aligned}
$$

The LO term clearly comes from $n_{3}^{m}(q)$, while NLO has contributions from $n_{2}^{m}(q)$ and $n_{4}^{m}(q)$. However, there is an additional complication as NLO will also come from $n_{3}^{m}$ at the next order (not shown above). More precisely, we need to determine

$$
D_{m}=\lim _{q \rightarrow \infty}\left[n_{3}^{m}-\frac{C_{2}}{q^{4}}\right] \frac{q^{6}}{\ln ^{3}(q)},
$$

where $C_{2}=\lim _{q \rightarrow \infty} q^{4} n^{m}(q)$, which is independent of $m$ as we discuss below. We find that $D_{m}$ is a nonzero constant that depends on the state $m$, which means that $C_{3}$ in $2 \mathrm{D}$ should be denoted $C_{3}^{m}$. Adding the NLO contributions from $n_{2}^{m}(q)$, $n_{3}^{m}(q)$, and $n_{4}^{m}(q)$, we find

$$
C_{3}^{0}=52.07 \text { and } C_{3}^{1}=1.01 .
$$

This $m$ dependence is absent in 3D for $\kappa_{*} \rightarrow 0$ but at the cost of the log-periodic term due to the Efimov effect [9]. That the present 2D case has state dependence is a result of the lack of 


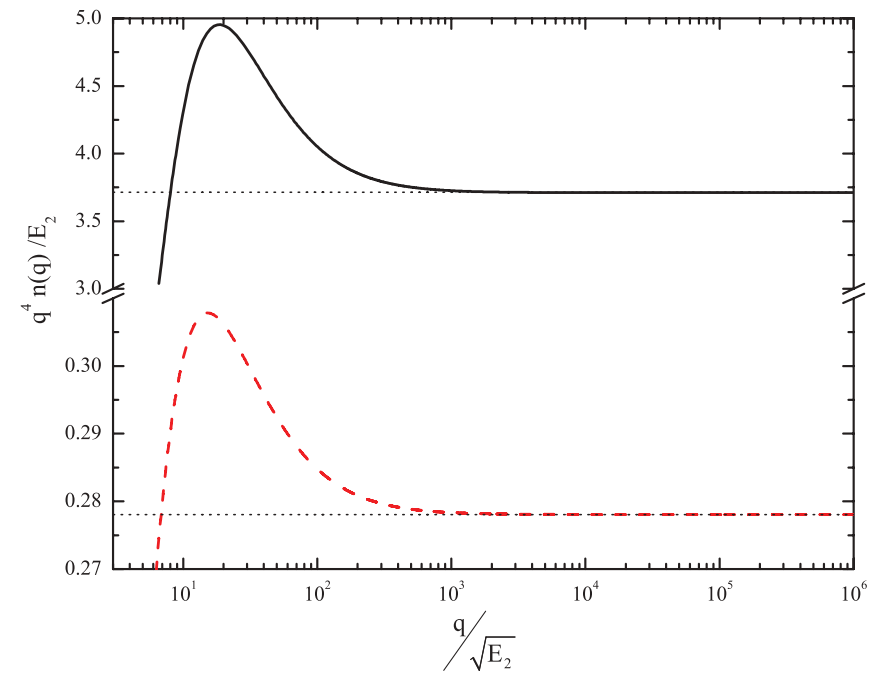

FIG. 1. (Color online) LO momentum distribution tail $q^{4} n(q)$ for ground (upper solid black line) and excited (lower dashed red line) three-body states. Note that the vertical axis is not uniform. The asymptotic dashed lines are the analytical results discussed in Appendix B.

geometric scaling symmetry in 2D. Note that the next order comes from $n_{1}^{m}(q)$ and differs by one power of $\ln (q)$ compared to the NLO term.

\section{A. Universal behavior}

The LO behavior in 2D is characterized by $C_{2}$. Explicitly, we have

$$
n_{3}^{0}(q) \rightarrow \frac{3.71 E_{2}}{q^{4}} \quad \text { and } \quad n_{3}^{1}(q) \rightarrow \frac{0.28 E_{2}}{q^{4}}
$$

These results have been obtained analytically (see Appendix B). We have also done a numerical check which is shown in Fig. 1. The units in Eq. (12) are, however, not natural in the same way that is seen in Eq. (8) where $\kappa_{*}$ provides the overall scale. The natural scale is $E_{3}$, and using this we find $3.71 E_{2} / 16.52 E_{2}=0.224$ and $0.28 E_{2} / 1.270 E_{2}=0.219$ for the ground and the excited state, respectively. This is a striking result that demonstrates the state independence of the LO term in $2 \mathrm{D}$ to within our numerical accuracy of $\sim 2 \%$. We thus predict that the two-body contact for a bosonic system in $2 \mathrm{D}$ with short-range attractive interactions is

$$
C_{2} / E_{3}=0.222 \pm 0.003
$$

where $E_{3}$ is the trimer energy. This should be compared to the relation $\frac{d E}{d \ln a}=\pi N C_{2}$ derived on general grounds in Ref. [17]. Here the factor $N$ appears due to our normalization which is different from Ref. [17]. We find agreement with this result within our numerical accuracy.

The universal tail behavior is far from trivial. In 3D and at unitarity, the discrete scale invariance induced by the divergence of the three-body problem, implies that the system should behave similarly irrespective of which trimer state one considers. This does not occur in 2D and the universal trimer energies are in some sense magic numbers multiplying the only scale available, $E_{2}$. Our results show that in spite of this major difference, the $2 \mathrm{D}$ momentum tail displays universal

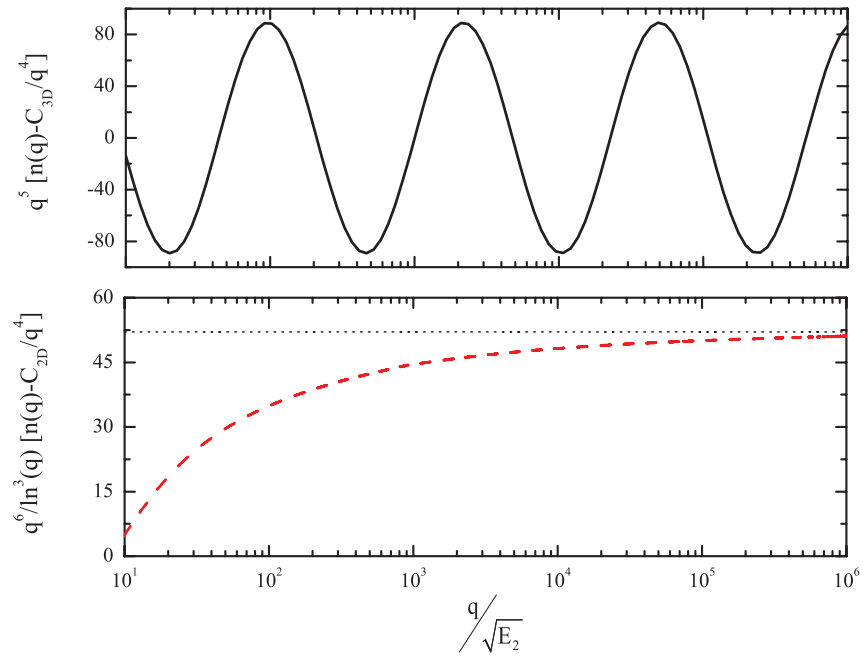

FIG. 2. (Color online) NLO momentum distribution comparison of 3D (upper panel) and 2D (lower panel). The 2D momentum distribution is the one of the ground state, but the result is similar for the excited state.

behavior, i.e., $C_{2} / E_{3}$ has the same value for both ground and excited states.

\section{DIMENSIONAL CROSSOVER}

Comparing the expressions in Eqs. (8) and (9), we see the same LO behavior at large momenta, but a vastly different NLO term. The oscillations seen in Eq. (8) can be traced directly to the discrete scaling symmetry, or more precisely, the breakdown of scale invariance in the system. It is known that the condition on the dimension $D$ for this behavior is $2.3<$ $D<3.8$ [29,30]. If we imagine an interpolation between 2D and $3 \mathrm{D}$, we would expect to see log-periodic terms in this range of $D$. The NLO term is therefore a telltale sign of effective dimensionality of the system as we will now discuss.

In experiments that study cold 2D quantum gases, one uses a tight transverse optical lattice potential to reduce the motion in this direction [1]. As recent experiments have beautifully demonstrated, the strength of the transverse optical lattice can be used to interpolate between 2D and $3 \mathrm{D}$ behavior of fermionic two-component systems $[14,16]$. Here we are concerned with bosonic systems, and our results above demonstrate how one can use the tail and, in particular, the NLO part of the momentum distribution as a measure of the effective dimensionality felt by the particles in the system by identifying the presence of log-periodic behavior. In Fig. 2 we show the extreme cases of 2D and 3D where the log-periodic oscillations are clearly seen in the latter, while the former has a smooth behavior.

A measurement of the overall functional form of the NLO term is thus enough to determine the effective dimensionality of the squeezed bosonic gas. In a real experiment, the motion in the transverse dimension is of course quantized by the lattice, and to get a full quantitative understanding this must be taken into account (see, for instance, Ref. [36]). However, since experiments have shown that it is possible to reach both the extreme $2 \mathrm{D}$ and the $3 \mathrm{D}$ regimes, there must necessarily be a dimensional crossover that can be seen in the NLO behavior. 
Of course, from a theoretical point of view it would be very attractive to be able to map the strength of the transverse confinement into some effective dimensionality $D_{\text {eff }}$ which could be noninteger [35].

\section{EXPERIMENTAL IMPLEMENTATION}

As we have demonstrated, the NLO term in the momentum distribution carries a telltale signature of the dimensionality of the quantum system under study. The 2D-3D crossover is of immense interest at the moment [14,16,36], and it has been shown that both the 3D and the strict 2D limits are accessible in experiment. Here we have discussed the crossover by using formalism applicable to either pure $2 \mathrm{D}$ or pure $3 \mathrm{D}$ without explicit consideration of the external confinement. Our results predict that a proof-of-principle experiment is possible by going to the two strict limits. However, the full crossover including the intermediate regime (quasi-2D) where the transverse confinement must be taken explicitly into account is experimentally addressable and should be explored theoretically in the future.

To connect our results directly to current experiments, we need to consider our units, the dimer binding energy $E_{2}$, and the effects of the transverse confinement on this two-body bound state. The interaction is controlled by Feshbach resonances [37]. However, under the confinement, the dimer energy is modified and becomes $E_{2}=B \hbar \omega_{z} \exp \left(-\sqrt{2 \pi} l_{z} /|a|\right) / \pi$ [38,39]. Here $\omega_{z}$ is the transverse harmonic confinement frequency, $l_{z}=\sqrt{\hbar / m \omega_{z}}$ is the trapping length, $a$ is the $3 \mathrm{D}$ scattering length associated with the Feshbach resonance, and $B=0.905$ is a constant. This formula holds for $a<0$ and $|a| \ll l_{z}$, while on resonance, $|a| \rightarrow \infty, E_{2}=0.244 \hbar \omega_{z}$. Corrections arise from the nonharmonic optical lattice [40], but they are not essential for our purposes. The dimer energy scale can be converted into a momentum scale $k_{0}$ through $E_{2}=$ $\hbar^{2} k_{0}^{2} / 2 m$. To access the tail behavior and the 2D-3D crossover, we see from Figs. 1 and 2 that the range $k \sim 10^{1}-10^{3} k_{0}$ is sufficient. Recent 2D Bose gas experiments [26,41] use $l_{z} \sim 3800 a_{0}$, where $a_{0}$ is the Bohr radius, which implies that $k_{0} \sim 10^{-4} a_{0}^{-1}$ when $|a|=\infty$. For the momentum distribution measurements [4,5], the maximum momentum reported is about $k \sim 10^{-3} a_{0}^{-1}$. This implies that an order of magnitude or two beyond the reported capabilities is necessary. However, if $a$ is tuned away from resonance to the $a<0$ side, $E_{2}$ will decrease rapidly according to the formulas above, inducing a corresponding rapid decrease of $k_{0}$ which should render the physics discussed here within reach of current experimental setups. Notice that the van der Waals length scale of about $100 a_{0}$ is in the deep tail, so there is no conflict with the universal zero-range description employed here.

\section{SUMMARY AND OUTLOOK}

We have taken a first step in the study of higher-order correlations and dimensional crossover by demonstrating how trimer observables in strongly interacting quantum gases can be used to probe dimensionality. Specifically, we see the breakdown of scale invariance directly in the functional form of the tail of the momentum distribution.
A clear direction for future study is a full inclusion of the transverse direction and the discrete spectrum it brings. We have shown that a crossover with fundamental influence on the momentum tail will happen, but mapping it out in a system that is squeezed by optical lattice(s) is the next task. This would also be interesting for the 1D-3D or 1D-2D crossovers. Another venue to explore is mass imbalanced systems where the spectrum is known to be richer in $2 \mathrm{D}$ than the equal mass case $[33,34]$.

\section{ACKNOWLEDGMENTS}

This work was partly support by funds provided by FAPESP (Fundação de Amparo à Pesquisa do Estado de São Paulo) and CNPq (Conselho Nacional de Desenvolvimento Científico e Tecnológico) of Brazil, and by the Danish Agency for Science, Technology, and Innovation.

\section{APPENDIX A: ASYMPTOTIC FORM OF $f(q)$}

Here we give the technical details of the analytical and numerical determination of the three-body wave function and momentum distributions. We will use units $\hbar=m=1$ and all energies are given in units of the two-body dimer energy $E_{2}$ implying that all momenta are in units of $\sqrt{E_{2}}$. In the symmetric case, where the three masses and the three two-body binding energies are set equal to one, the spectator function fulfills the integral equation

$$
f(q)=\frac{1 / \pi}{\ln \left(\sqrt{E_{3}+3 / 4 q^{2}}\right)} \int d^{2} k \frac{f(k)}{E_{3}+q^{2}+k^{2}+\mathbf{k} \cdot \mathbf{q}} .
$$

This can be cast into the useful form

$$
f(q)=\frac{\alpha\left(q, E_{3}\right)}{\pi} \int_{0}^{\infty} d k \frac{k f(k)}{E_{3}+q^{2}+k^{2}} \int_{0}^{2 \pi} \frac{d \theta}{1+a \cos \theta},
$$

with $a=k q /\left(E_{3}+q^{2}+k^{2}\right)$ and where we have defined

$$
\alpha\left(q, E_{3}\right)=\frac{1}{\ln \left(\sqrt{E_{3}+3 / 4 q^{2}}\right)} .
$$

The angular integral is

$$
\int_{0}^{2 \pi} \frac{d \theta}{1+a \cos \theta}=\frac{2 \pi}{\sqrt{1-a^{2}}} \text { for } 0<a<1
$$

and one obtains

$$
f(q)=2 \alpha\left(q, E_{3}\right) \int_{0}^{\infty} d k \frac{k f(k)}{\left(E_{3}+q^{2}+k^{2}\right) \sqrt{1-\frac{q^{2} k^{2}}{\left(E_{3}+q^{2}+k^{2}\right)^{2}}}}
$$

which can be rewritten as

$$
\begin{aligned}
f(q)= & 2 \alpha\left(q, E_{3}\right)\left[\int_{0}^{\Lambda} d k \frac{k f(k)}{\left(E_{3}+q^{2}+k^{2}\right) \sqrt{1-\frac{q^{2} k^{2}}{\left(E_{3}+q^{2}+k^{2}\right)^{2}}}}\right. \\
& \left.\left.+\int_{\Lambda}^{\infty} d k \frac{k f(k)}{\left(E_{3}+q^{2}+k^{2}\right) \sqrt{1-\frac{q^{2} k^{2}}{\left(E_{3}+q^{2}+k^{2}\right)^{2}}}}\right], \quad \text { (A6 }\right)
\end{aligned}
$$


where $\Lambda$ is a large-momentum cutoff that will be useful below. Taking $\Lambda \gg \sqrt{E_{3}}$, the spectator function in Eq. (A6) is approximately given by

$$
\begin{aligned}
f(q) \approx & 2 \alpha\left(q, E_{3}\right) \int_{0}^{\Lambda} d k \frac{k f(k)}{\left(E_{3}+q^{2}+k^{2}\right) \sqrt{1-\frac{q^{2} k^{2}}{\left(E_{3}+q^{2}+k^{2}\right)^{2}}}} \\
& +2 \alpha\left(q, E_{3}\right) \int_{\Lambda}^{\infty} d k \frac{k f(k)}{\left(q^{2}+k^{2}\right) \sqrt{1-\frac{q^{2} k^{2}}{\left(q^{2}+k^{2}\right)^{2}}}} .
\end{aligned}
$$

For $q \rightarrow \infty$, the first term on the right-hand side of Eq. (A7) tends to zero in the following manner:

$$
f_{1}(q) \approx \frac{2}{q^{2} \ln q} \int_{0}^{\Lambda} d k \frac{k f(k)}{\sqrt{1-\frac{q^{2} k^{2}}{\left(q^{2}+k^{2}\right)^{2}}}}
$$

Now, we assume that

$$
f(q) \underset{q \rightarrow \infty}{\rightarrow} \frac{\ln q}{q^{2}} .
$$

Inserting this ansatz and taking the limit $q \rightarrow \infty$ in the second term on the right-hand side of Eq. (A7) one finds

$$
\begin{aligned}
f_{2}(q) & \approx \frac{2}{\ln q} \int_{\Lambda}^{\infty} d k \frac{k \ln k}{k^{2}\left(q^{2}+k^{2}\right) \sqrt{1-\frac{q^{2} k^{2}}{\left(q^{2}+k^{2}\right)^{2}}}} \\
& \rightarrow \frac{2}{\ln q} \int_{\Lambda}^{\infty} d k \frac{\ln k}{k\left(q^{2}+k^{2}\right)},
\end{aligned}
$$

when $q \rightarrow \infty$. Changing variables to $y=k / q$, the second spectator function term becomes

$$
f_{2}(q) \approx \frac{2}{q^{2} \ln q} \int_{\Lambda / q}^{\infty} \frac{d y}{y} \frac{\ln y+\ln q}{\left(1+y^{2}\right)}
$$

which can be split into

$$
\begin{aligned}
f_{2}(q) \approx & \frac{2}{q^{2} \ln q}\left[\int_{\Lambda / q}^{\infty} \frac{d y}{y} \frac{\ln y}{\left(1+y^{2}\right)}\right. \\
& \left.+\ln q \int_{\Lambda / q}^{\infty} \frac{d y}{y} \frac{1}{\left(1+y^{2}\right)}\right]
\end{aligned}
$$

The first integral term on the right-hand side of Eq. (A12) is

$$
\begin{aligned}
\int_{\Lambda / q}^{\infty} \frac{d y}{y} \frac{\ln y}{\left(1+y^{2}\right)} & =\left.\frac{1}{2} \frac{\ln ^{2} y}{1+y^{2}}\right|_{\Lambda / q} ^{\infty}+\int_{\Lambda / q}^{\infty} d y \frac{y \ln ^{2} y}{\left(1+y^{2}\right)^{2}} \\
& \rightarrow-\frac{1}{2} \ln ^{2} \frac{\Lambda}{q}=-\frac{1}{2} \ln ^{2} q
\end{aligned}
$$

for $q \rightarrow \infty$. The second term on the right-hand side of Eq. (A12) is

$$
\begin{aligned}
\int_{\Lambda / q}^{\infty} \frac{d y}{y} \frac{1}{\left(1+y^{2}\right)} & =\left.\frac{\ln y}{1+y^{2}}\right|_{\Lambda / q} ^{\infty}+2 \int_{\Lambda / q}^{\infty} d y \frac{y \ln y}{\left(1+y^{2}\right)^{2}} \\
& \rightarrow-\ln \frac{\Lambda}{q}=\ln q
\end{aligned}
$$

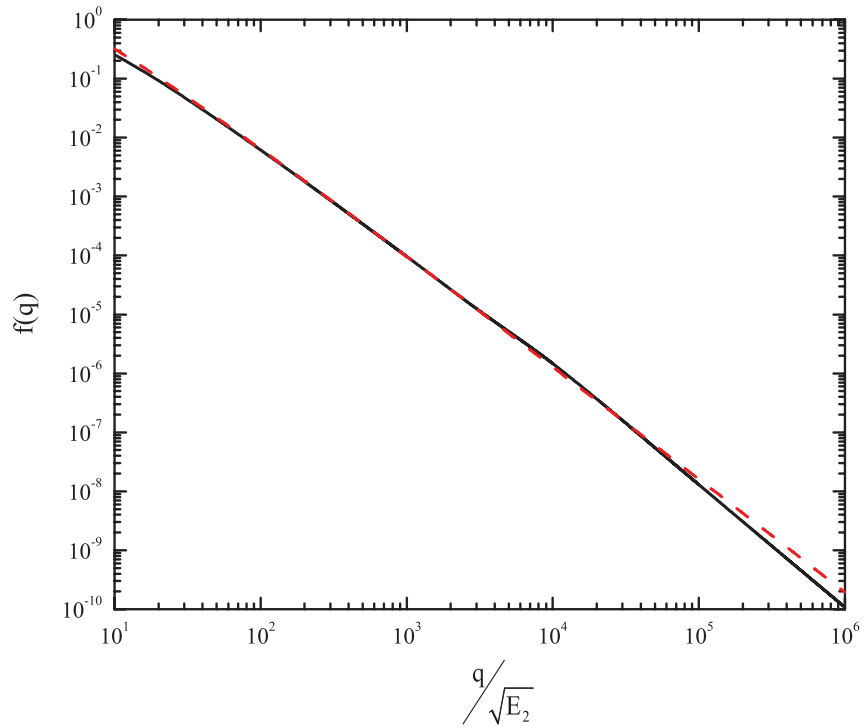

FIG. 3. (Color online) Spectator function $f(q)$ for the ground state calculated numerically (black solid line) and using the ansatz $f(q)=A_{0} \frac{\ln q}{q^{2}}$ (red dashed line). The solid (black) line tends to oscillate around the dashed (red) one as $q \rightarrow \infty$ due to finite numerical precision.

for $q \rightarrow \infty$. Inserting the results of Eqs. (A13) and (A14) in Eq. (A11) we arrive at

$$
f_{2}(q) \approx \frac{2}{q^{2} \ln q}\left(\ln ^{2} q-\frac{1}{2} \ln ^{2} q\right)=\frac{\ln q}{q^{2}}
$$

Collecting the results Eqs. (A8) and (A15) we conclude that the ansatz in Eq. (A9) gives us the asymptotic behavior of the exact spectator function.

In Fig. 3 both the spectator function obtained from the numerical solution of the set of coupled integral equations and the spectator function asymptotic behavior given in Eq. (A9) for the ground state are shown. The log-log scale shows us that both the magnitude and the line's inclination are very close in the region of $q$ between 100 and 2000 for the numerical and analytical calculations. For $q>2000$, the curve which represents the numerical solution of the integral equations starts to oscillate around the analytical form.

\section{APPENDIX B: MOMENTUM DENSITY ASYMPTOTIC BEHAVIOR}

The one-body momentum distribution is given by

$n(q)=\int d^{2} p\left|\frac{f(q)+f\left(\left|\mathbf{p}-\frac{\mathbf{q}}{2}\right|\right)+f\left(\left|\mathbf{p}+\frac{\mathbf{q}}{2}\right|\right)}{E_{3}+\mathbf{p}^{2}+\frac{3}{4} \mathbf{q}^{2}}\right|^{2}$,

and can be split into four parts through

$$
n^{m}(q)=\sum_{l=1}^{4} n_{l}^{m}(q)
$$


where superscript $m$ distinguishes ground $(m=0)$ and excited states $(m>0)$. The individual expressions are

$$
\begin{gathered}
n_{1}^{m}(q)=f_{m}(q)^{2} \int d^{2} p \frac{1}{\left(E_{3}^{m}+\mathbf{p}^{2}+\frac{3}{4} \mathbf{q}^{2}\right)^{2}}=\frac{\pi f_{m}(q)^{2}}{E_{3}^{m}+\frac{3}{4} \mathbf{q}^{2}} \\
n_{2}^{m}(q)=4 f_{m}(q) \int d^{2} p \frac{f_{m}\left(\left|\mathbf{p}+\frac{\mathbf{q}}{2}\right|\right)}{\left(E_{3}^{m}+\mathbf{p}^{2}+\frac{3}{4} \mathbf{q}^{2}\right)^{2}} \\
=4 f_{m}(q) \int d^{2} k \frac{f_{m}(k)}{\left(E_{3}^{m}+\mathbf{k}^{2}+\mathbf{q}^{2}+\mathbf{k} \cdot \mathbf{q}\right)^{2}} \\
n_{3}^{m}(q)=2 \int d^{2} p \frac{f^{2}\left(\left|\mathbf{p}+\frac{\mathbf{q}}{2}\right|\right)}{\left(E_{3}^{m}+\mathbf{p}^{2}+\frac{3}{4} \mathbf{q}^{2}\right)^{2}} \\
=2 \int d^{2} k \frac{\left(f_{m}(k)\right)^{2}}{\left(E_{3}^{m}+\mathbf{k}^{2}+\mathbf{q}^{2}+\mathbf{k} \cdot \mathbf{q}\right)^{2}} \\
n_{4}^{m}(q)=2 \int d^{2} p \frac{f_{m}\left(\left|\mathbf{p}+\frac{\mathbf{q}}{2}\right|\right) f_{m}\left(\left|\mathbf{p}-\frac{\mathbf{q}}{2}\right|\right)}{\left(E_{3}^{m}+\mathbf{p}^{2}+\frac{3}{4} \mathbf{q}^{2}\right)^{2}} \\
=2 \int d^{2} k \frac{f_{m}(k) f_{m}(|\mathbf{k}-\mathbf{q}|)}{\left(E_{3}^{m}+\mathbf{k}^{2}+\mathbf{q}^{2}+\mathbf{k} \cdot \mathbf{q}\right)^{2}}
\end{gathered}
$$

The subscript index on $f_{m}(q)$ indicates the state under consideration. Here we are interested in the limit $q \rightarrow \infty$, where we find

$$
\begin{gathered}
n_{1}^{m}(q) \approx \frac{4 \pi}{3} \frac{\left(f_{m}(q)\right)^{2}}{q^{2}} \\
n_{2}^{m}(q) \approx \frac{4 \pi}{q^{2}}\left(f_{m}(q)\right)^{2} \ln \left(\sqrt{E_{3}^{m}+\frac{3}{4} \mathbf{q}^{2}}\right) \approx \frac{4 \pi}{q^{2}}\left(f_{m}(q)\right)^{2} \ln (q), \\
n_{3}^{m}(q) \approx \frac{4 \pi}{q^{4}} \int_{0}^{\infty} d k k\left(f_{m}(k)\right)^{2} \\
n_{4}^{m}(q) \approx \frac{2 \pi}{q^{2}}\left(f_{m}(q)\right)^{2} \ln \left(\sqrt{E_{3}^{m}+\frac{3}{4} \mathbf{q}^{2}}\right) \approx \frac{2 \pi}{q^{2}}\left(f_{m}(q)\right)^{2} \ln (q) .
\end{gathered}
$$

The asymptotic form of $f(q)$ when $q \rightarrow \infty$ is (see derivation in Appendix A)

$$
f_{m}(q) \underset{q \rightarrow \infty}{\rightarrow} A_{m} \frac{\ln q}{q^{2}} .
$$

The functional form of asymptotic behavior is the same for both ground and excited states. However, the normalization constant

$$
A_{m}=\lim _{q \rightarrow \infty} f_{m}(q) \frac{q^{2}}{\ln q}
$$

is different. Since we are using the normalization $\int d^{2} k n(k)=$ 1 , one finds

$$
A_{0} \approx 1.800 \text { and } A_{1} \approx 0.251 .
$$

The numerical results for the asymptotics are shown in Figs. 4 and 5.

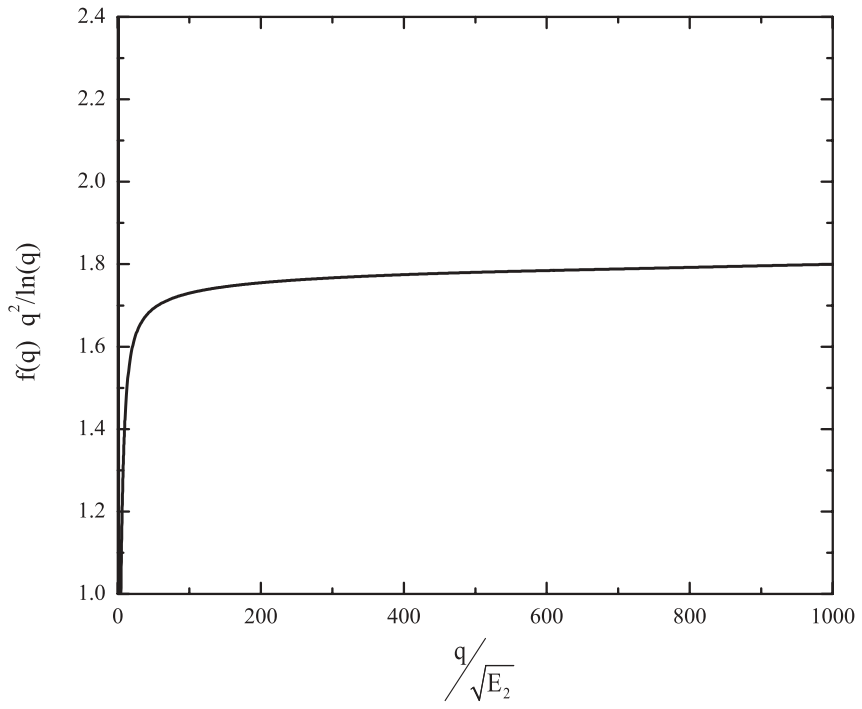

FIG. 4. Asymptotic behavior of $f(q)$ for the ground state.

Inserting Eq. (B11) in Eqs. (B7)-(B10) one obtains the normalized asymptotic behavior

$$
\begin{aligned}
& n_{1}^{m}(q) \rightarrow A_{m}^{2} \frac{4 \pi}{3} \frac{\ln ^{2}(q)}{q^{6}}, \\
& n_{2}^{m}(q) \rightarrow A_{m}^{2} 4 \pi \frac{\ln ^{3}(q)}{q^{6}}, \\
& n_{3}^{m}(q) \rightarrow \frac{C_{m}}{q^{4}} \text { with } C_{m}=\int_{0}^{\infty} d k k\left(f_{m}(k)\right)^{2}, \\
& n_{4}^{m}(q) \rightarrow A_{m}^{2} 2 \pi \frac{\ln ^{3}(q)}{q^{6}} .
\end{aligned}
$$

The normalization constants given in Eq. (B13) determine the asymptotic values and behaviors of the partial momentum density in Eqs. (B14)-(B17) when $q \rightarrow \infty$.

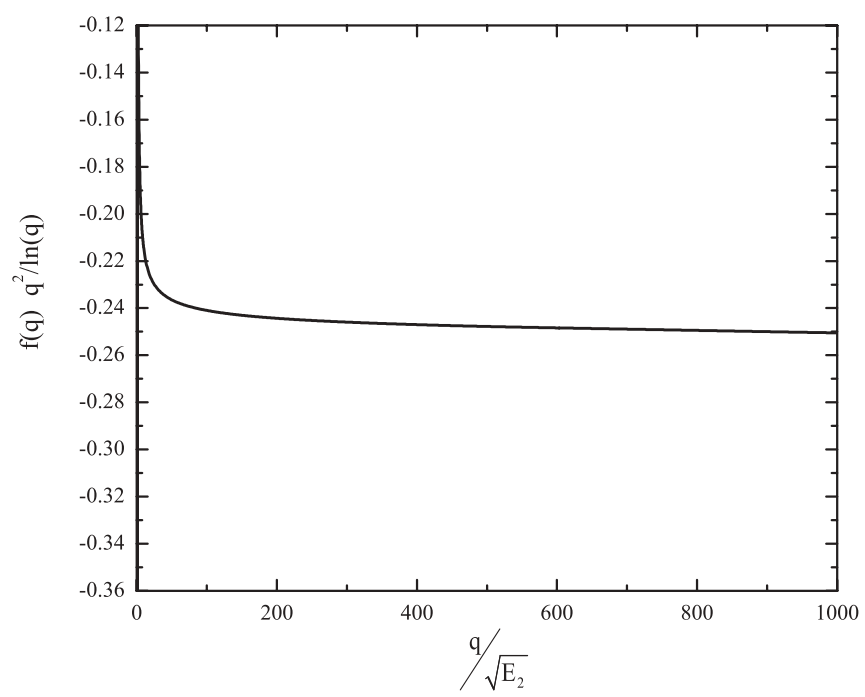

FIG. 5. Same as Fig. 4 for the first excited state. 
[1] I. Bloch, J. Dalibard, and W. Zwerger, Rev. Mod. Phys. 80, 885 (2008).

[2] S. Tan, Ann. Phys. 323, 2952 (2008); 323, 2971 (2008); 323, 2987 (2008).

[3] M. Olshanii and V. Dunjko, Phys. Rev. Lett. 91, 090401 (2003).

[4] J. T. Stewart, J. P. Gaebler, T. E. Drake, and D. S. Jin, Phys. Rev. Lett. 104, 235301 (2010).

[5] E. D. Kuhnle, H. Hu, X. J. Liu, P. Dyke, M. Mark, P. D. Drummond, P. Hannaford, and C. J. Vale, Phys. Rev. Lett. 105, 070402 (2010).

[6] R. Combescot, F. Alzetto, and X. Leyronas, Phys. Rev. A 79, 053640 (2009).

[7] A. M. J. Schakel, arXiv:1007.3452v1.

[8] E. Braaten, D. Kang, and L. Platter, Phys. Rev. Lett. 106, 153005 (2011).

[9] Y. Castin and F. Werner, Phys. Rev. A 83, 063614 (2011).

[10] F. Werner and Y. Castin, Phys. Rev. A 86, 053633 (2012).

[11] R. J. Wild, P. Makotyn, J. M. Pino, E. A. Cornell, and D. S. Jin, Phys. Rev. Lett. 108, 145305 (2012).

[12] K. Martiyanov, V. Makhalov, and A. Turlapov, Phys. Rev. Lett. 105, 030404 (2010).

[13] B. Fröhlich, M. Feld, E. Vogt, M. Koschorreck, W. Zwerger, and M. Kohl, Phys. Rev. Lett. 106, 105301 (2011).

[14] P. Dyke, E. D. Kuhnle, S. Whitlock, H. Hu, M. Mark, S. Hoinka, M. Lingham, P. Hannaford, and C. J. Vale, Phys. Rev. Lett. 106, 105304 (2011).

[15] M. Feld, B. Fröhlich, E. Vogt, M. Koschorreck, and M. Köhl, Nature (London) 480, 75 (2011).

[16] A. T. Sommer, L. W. Cheuk, M. J. H. Ku, W. S. Bakr, and M. W. Zwierlein, Phys. Rev. Lett. 108, 045302 (2012).

[17] F. Werner and Y. Castin, arXiv:1001.0774v1; Phys. Rev. A 86, 013626 (2012).

[18] M. Valiente, N. T. Zinner, and K. Mølmer, Phys. Rev. A 84, 063626 (2011).

[19] C. Langmack, M. Barth, W. Zwerger, and E. Braaten, Phys. Rev. Lett. 108, 060402 (2012).

[20] B. Fröhlich, M. Feld, E. Vogt, M. Koschorreck, M. Köhl, C. Berthod, and T. Giamarchi, Phys. Rev. Lett. 109, 130403 (2012).
[21] E. Vogt, M. Feld, B. Frohlich, D. Pertot, M. Koschorreck, and M. Kohl, Phys. Rev. Lett. 108, 070404 (2012).

[22] M. Olshanii, H. Perrin, and V. Lorent, Phys. Rev. Lett. 105, 095302 (2010).

[23] J. Hofmann, Phys. Rev. Lett. 108, 185303 (2012).

[24] E. Taylor and M. Randeria, Phys. Rev. Lett. 109, 135301 (2012).

[25] L. P. Pitaevskii and A. Rosch, Phys. Rev. A 55, R853 (1997).

[26] C.-L. Hung, X. Zhang, N. Gemelke, and C. Chin, Nature (London) 470, 236 (2011).

[27] L. W. Bruch and J. A. Tjon, Phys. Rev. A 19, 425 (1979).

[28] S. K. Adhikari, A. Delfino, T. Frederico, I. D. Goldman, and L. Tomio, Phys. Rev. A 37, 3666 (1988); S. K. Adhikari, A. Delfino, T. Frederico, and L. Tomio, ibid. 47, 1093 (1993).

[29] E. Nielsen, D. V. Fedorov, and A. S. Jensen, Phys. Rev. A 56, 3287 (1997).

[30] E. Nielsen, D. V. Fedorov, A. S. Jensen, and E. Garrido, Phys. Rep. 347, 373 (2001).

[31] V. Efimov, Yad. Fiz. 12, 1080 (1970); Sov. J. Nucl. Phys. 12, 589 (1971).

[32] E. Braaten and H. W. Hammer, Phys. Rep. 428, 259 (2006).

[33] F. Bellotti, T. Frederico, M. T. Yamashita, D. V. Fedorov, A. S. Jensen, and N. T. Zinner, J. Phys. B 44, 205302 (2011).

[34] F. Bellotti, T. Frederico, M. T. Yamashita, D. V. Fedorov, A. S. Jensen, and N. T. Zinner, Phys. Rev. A 85, 025601 (2012).

[35] M. Valiente, N. T. Zinner, and K. Mølmer, Phys. Rev. A 86, 043616 (2012).

[36] S. K. Baur, B. Frohlich, M. Feld, E. Vogt, D. Pertot, M. Koschorreck, and M. Kohl, Phys. Rev. A 85, 061604(R) (2012).

[37] C. Chin, R. Grimm, P. S. Julienne, and E. Tiesinga, Rev. Mod. Phys. 82, 1225 (2010).

[38] D. S. Petrov and G. V. Shlyapnikov, Phys. Rev. A 64, 012706 (2001)

[39] L. Pricoupenko and M. Olshanii, J. Phys. B 40, 2065 (2007).

[40] G. Orso, L. P. Pitaevskii, S. Stringari, and M. Wouters, Phys. Rev. Lett. 95, 060402 (2005).

[41] T. Yefsah, R. Desbuquois, L. Chomaz, K. J. Günter, and J. Dalibard, Phys. Rev. Lett. 107, 130401 (2011). 